\title{
Wave Height Distribution of Bimodal Spectral Wave based on Simulated Wave Elevation
}

\author{
Xiaolin Huan ${ }^{1,2}$, Shifeng Xue ${ }^{1}$, Xuejun Fan ${ }^{3}$ and Changliang $\mathbf{L i}^{4, * *}$ \\ ${ }^{I}$ College of Pipeline and Civil Engineering, China University of Petroleum, Qingdao 266580, China \\ ${ }^{2}$ College of Civil Engineering and Architecture, Shandong University of Science and Technology, Qingdao 266590, China \\ ${ }^{3}$ Department of Mechanical Engineering, Lamar University, Texas, PO 10028, United States \\ ${ }^{4}$ School of Petroleum Engineering, China University of Petroleum, Qingdao 266580, China
}

Received 1 September 2020; Accepted 29 November 2020

\begin{abstract}
The selection of the design wave height in ocean engineering is greatly related to the wave height distribution in the sea area. Many existing empirical and theoretical distribution formulae of wave height are linear or nonlinear and suitable only for narrow spectrum based on the classic Rayleigh distribution. However, these formulae cannot be effectively applied due to several problems, such as complex forms or difficult determination of key parameters. To explore the influence of mixed sea states (with bimodal spectrum) and nonlinearity on the wave height distribution in the actual ocean environment, this study proposed a new Weibull distribution model of wave height that included the effects of spectral width and nonlinearity. By analyzing the characteristics of each parameter of the Weibull distribution, the relationship between the shape and scale parameters was determined. The wave duration of different types of bimodal spectra was simulated through the Monte Carlo simulation method, and the influence of different spectral types and nonlinearity on the wave height distribution was investigated. The calculation formula of the shape coefficient of the Weibull distribution was obtained through fitting. Results demonstrate that the spectral width has minimal influence on the wave height distribution under deep water condition, and the wave height follows the Rayleigh distribution. For swell-dominated sea state, the extreme wave height can reach two times that obtained using the Rayleigh distribution when the nonlinearity is large. The new Weibull model that considers the spectral width and nonlinear factors can accurately describe the wave height distribution of mixed sea states. The proposed method provides a good prospect to the selection of design wave height in complex sea conditions.
\end{abstract}

Keywords: Bimodal spectrum, Wave height distribution, Nonlinear, Spectral width, Weibull distribution, Shape parameter

\section{Introduction}

The design wave height is one of the critical factors that must be considered in the design of coastal and ocean structures. This factor is necessary in estimating the safety and cost of engineering structures under severe sea conditions. Design wave heights usually include average, maximum, significant or $1 / \mathrm{n}$ average wave heights. These heights are selected from irregular wave chains and are related to each other. When deriving various representative wave height relationships theoretically, the first step is to identify the mathematical model of the wave height distribution. Scholars performed numerous research on the distribution of wave height. According to the linear model of random waves, the most widely used wave height distribution is the Rayleigh distribution formula, which is theoretically derived from the Longuet-Higgins equation under the assumption of linearity and narrow spectrum [1].

However, the statistics of the sea wave observation data show that the opportunities for pure wind waves and pure swells are relatively scarce, and most of them appear in the form of mixed waves. Mixed waves refer to situations where wind waves and swells exist on the sea at the same time or when the wind direction changes suddenly. The spectrum

*E-mail address: fifalc1@163.com

ISSN: 1791-2377 @ 2020 School of Science, IHU. All rights reserved. doi:10.25103/iestr.136.06 structure of mixed waves is highly complex and diverse. Except for few cases where the spectrum is unimodal, most spectra are bimodal or multimodal. The wave spectrum is neither a narrowband spectrum nor a Gaussian random process and often exhibits nonlinear properties. Therefore, the Rayleigh distribution often leads to the underestimation or overestimation of the design wave height. The former case will result in safety hazards to the marine engineering structure and cause the structure to be damaged by excessive wave force.

Numerous related studies on the characteristics of actual ocean waves were conducted. Most of these works analyzed the effect of nonlinearity or the spectral width of single-peak spectrum on the wave height distribution. The joint distribution of the wave height and period of the bimodal spectrum was also investigated. However, these studies did not consider the coupling effect of the bimodal spectrum and nonlinearity on the wave height distribution. Therefore, determining an easy-to-apply formula that reveals the distribution of wave heights and consider the spectral width and nonlinearity is an urgent concern that must be solved.

Many optimization problems involve multiple objectives. For the network-clustering problem, many multi-objective optimization models have been proposed. Pizzuti [10] presented a multi-objective genetic algorithm to uncover communities in networks. Recently, Gong et al. [11] proposed a multi-objective discrete particle swarm optimization algorithm for network clustering. Experiments 
have demonstrated that the proposed algorithm is very effective.

On the basis of the above analysis, this study determined the key parameters of Weibull distribution through analysis and simulated second-order nonlinear waves by using the Monte Carlo method. The objective of this study is to investigate the influence of spectral width and nonlinearity on wave height distribution and provide reference to engineering designers.

\section{State of the art}

The distribution of wave height or joint distribution of wave height and period is extensively investigated. Kvingedal analyzed the distribution of individual wave and crest heights by using the 14-year data collected at a certain point in the northern North Sea and compared them through known theoretical distribution formulae. The Rayleigh distribution is conservative in the estimation of wave heights, but the results are not universally applicable [7]. Mackay used three methods to analyze the long-term distribution of an individual wave and compared and analyzed the deviation caused by the tail shape of the extreme significant wave height distribution during a storm. However, the effect of wave nonlinearity was not considered [8]. The nonlinearity of random waves was analyzed by Tayfun [9], but the analysis only focused on narrow-band spectrum theory without considering the influence of wide spectrum. On the basis of the inverse scattering transformation solution of the third-order nonlinear Schrodinger (NLS) equation, Zhang et al. theoretically derived a method to calculate the eigenvalue of the NLS equation from the time history data of the measured wave height. Nevertheless, the mixed wave factor was not considered [10]. Forristall explored the distribution of wave crest height on the basis of the second-order nonlinearity. The findings revealed that the wave height deviates from the Rayleigh distribution as the level of nonlinearity increases. However, this study did not explore the influence of spectral width on the wave height distribution [11]. Feng investigated the nonlinear probability distribution of wave height by using the high-order spectral numerical model. The results suggested that the distribution conforms to the Rayleigh distribution when the significant wave height is relatively small. As the wave nonlinearity and significant wave height increase, the deviation of the wave height distribution from the Rayleigh distribution increases. In addition, the model is relatively complex and difficult to apply [12]. Haselsteiner found that the exponential Weibull distribution can effectively characterize the actual wave height distribution. However, this analysis did not involve influence factors, such as wave nonlinearity and spectral width [13]. Based on the nonlinear shallow water wave function, $\mathrm{Li}$ calculated the interval distribution of wave height and established a statistical time-domain analysis model to estimate such distribution. However, the influence of nonlinearity on the wave height distribution is not analyzed [14]. Rifat Tur used the classical linear and intelligent nonlinear time series methods to predict the shortterm maximum wave height to effectively improve the accuracy of wave height prediction. However, the presented approach is complex and difficult to popularize and apply [15].

Substantial effort has been devoted to the study on bimodal waves in the past decades. Chen et al. evaluated the applicability of the Ochi-Hubble bimodal spectrum to sea areas near islands and reefs and obtained the parameter fitting formula of the wind wave and swell components. However, the formula is only applicable to the South China Sea [16]. Stephen studied the influence of different percentages of swell energy in the energy-conserving bimodal spectrum wave on the wave height distribution near the wave maker and in shallow water. The analysis require verification through measured data [17]. Guan et al. explained the overestimation problem of the high-frequency part of the double-peak ocean wave spectrum and proposed an iterative algorithm as a solution [18]. Forristall analyzed the joint statistical properties of wave height and period on the basis of linear simulation and inferred that such a simulation can predict the maximum wave with sufficient accuracy as long as the frequency difference between the two spectral peaks of the bimodal spectrum is not excessively large. However, the effect of nonlinear factors in shallow water was not considered [19]. Didenkulova conducted a direct numerical simulation of the evolution of irregular nonlinear waves by using bimodal spectrum and studied the influence of the coexistence of additional wave systems on the statistical characteristics and spectral patterns of waves. However, the distribution rules of the spectral patterns and statistical wave heights were not revealed [20]. Huang proposed a binary lognormal mixed model to characterize the joint distribution of the wave height and period of sea conditions with double-peak spectrum characteristics. This model can accurately describe the bimodal properties of the spectrum but does not consider the effect of the spectrum width of the bimodal spectrum on the distribution of wave height [21]. James $\mathrm{Hu}$ discussed the influence of single-peak spectral width and nonlinearity on wave height distribution under deep-water conditions but disregarded the case of limited water depth, especially shallow water [22]. Li et al. investigated the influence of spectral width and nonlinearity on wave height distribution under a single-peak spectrum but did not consider the case of mixed waves [23]. Martell-Dubois used the wave data collected near the Caribbean Coast of Mexico to discuss the frequency and directional structure of the wave field generated during the passage of three hurricanes. The relationship between the translational velocity of the hurricanes and wave group velocity can explain whether a spectral bi-modularity is present in the frequency and direction [24]. Alessandro Toffoli used the second-order wave model to study the influence of unimodal and bimodal spectra on the statistical characteristics of waves under different water depth conditions and concluded that the nonlinear interaction is the strongest when the two spectra are slightly separated in direction. However, the influence of spectral width on the wave height distribution was not discussed [25].

Most of the existing studies focused on the influence of wave nonlinearity on the joint distribution of wave height and period, as well as on the effect of the spectral width of the unimodal spectrum and the spectral type of the bimodal spectrum on the wave height distribution. However, the coupling effects of wave nonlinearity and width of the bimodal spectrum on the distribution of wave height are rarely studied. In the present study, a widely used Weibull distribution model that can flexibly select parameters was adopted. The relationship between shape and scale parameters was analyzed, and the former was established as the key factor. The second-order nonlinear irregular wave was simulated using the Monte Carlo method, and the influence of the bimodal spectral width and wave 
nonlinearity on the wave height distribution was discussed. The least squares method was applied to obtain the calculation formula of the shape parameter, which is associated with the spectral width and nonlinearity. The findings could provide a reference for the prediction of the design wave height.

The remainder of this study is organized as follows. In section 3 , the relationship between the two main parameters of the Weibull distribution is deduced. The bimodal spectrum type used for simulation is selected, and the formulae for the second-order nonlinear wave simulation are described. Section 4 analyzes the effects of spectral width and nonlinearity on the distribution of wave height and verifies the calculation formula of the shape parameter. Section 5 summarizes the findings and provides the relevant conclusions.

\section{Methodology}

\subsection{Relationship between the two main parameters of the} Weibull distribution

Longuet-Higgins demonstrated that the Rayleigh distribution is applicable to the heights of waves in the sea. The two main assumptions for the theoretical derivation state that (1) the frequency spectrum consists of a single narrow frequency band and (2) the waves can be considered as the sum of a large number of contributions with the same frequency and random phase. The Rayleigh probability density function (PDF) can be expressed as

$$
p_{H}(h)=\frac{4 h}{H_{s}^{2}} \exp \left[-2\left(\frac{h}{H_{s}}\right)^{2}\right], h \geq 0
$$

where $h$ denotes the wave height and $H_{s}$ is the significant wave height.

The Rayleigh distribution is consistent with the waves in deep water. However, as the water depth decreases, the distribution of the measured wave height gradually deviates from the Rayleigh distribution. Given that the actual spectrum is not always narrow, scholars use the Weibull distribution to describe the wave height distribution. The Weibull model has no theoretical advantage in the analysis of the wave height distribution, but it is highly flexible in practical applications and consistent with the actual spectrum. The cumulative PDF of the Weibull distribution can be written as

$$
P_{H}(h)=1-\exp \left[-\left(\frac{h-\xi}{\theta}\right)^{\alpha}\right], h \geq \xi
$$

where $\xi, \theta$, and $\alpha$ represent the location, scale and shape parameters, respectively.

Equation (2) becomes a Rayleigh distribution when $\alpha=2$ and $\xi=0$.In this study, $\xi$ is always set to zero. For convenience of engineering application, $\theta$ might be replaced with $\beta H_{s}$. The probability of the density function of the corresponding Weibull distribution can be expressed as

$$
p_{H}(h)=\frac{\alpha h^{\alpha-1}}{\left(\beta H_{s}\right)^{\alpha}} \exp \left[-\left(\frac{h}{\beta H_{s}}\right)^{\alpha}\right], h \geq 0
$$

The derivation for the $1 / \mathrm{n}$ wave height based on the Weibull wave height distribution is explained below. The probability of exceedance associated with Eq. (3) is written as

$Q_{H}(h)=1-P_{H}(h)=\exp \left[-\left(\frac{h}{\beta H_{s}}\right)^{\alpha}\right], h \geq 0$

By denoting the wave height that corresponds to $Q=1 / n$ as $\hat{H}_{1 / n}$, the above expression becomes

$\frac{1}{\mathrm{n}}=\exp \left[-\left(\frac{\tilde{H}_{1 / n}}{\beta H_{s}}\right)^{\alpha}\right]$

By rearranging the terms, Eq. (5) can be expressed as

$\tilde{H}_{1 / n}=[\ln (n)]^{\frac{1}{\alpha}} \beta H_{s}$

In accordance with the definition of the $1 / \mathrm{nth}$ average wave height

$H_{1 / n}=n \int_{\hat{H}_{1 / n}}^{\infty} \frac{\alpha h^{\alpha}}{\left(\beta H_{s}\right)^{\alpha}} \exp \left[-\left(\frac{h}{\beta H_{s}}\right)^{\alpha}\right] d h$

or

$\frac{H_{1 / n}}{\beta H_{s}}=n \int_{[\ln (n)]^{\frac{1}{\alpha}}}^{\infty} \alpha h^{\alpha} \exp \left(-h^{\alpha}\right) d h$

If $n=3$, Eq. (8) can be rewritten as

$\beta=1 /\left\{3 \int_{[\ln (3)]^{\frac{1}{\alpha}}}^{\infty} \alpha h^{\alpha} \exp \left(-h^{\alpha}\right) d h\right\}$

If $\alpha$ is known, the Weibull PDF can be determined. Therefore, the determination of the appropriate value of $\alpha$ must be realized.

\subsection{Inputted bimodal spectral type}

The bimodal spectrum can be classified into three main types. The first type is mixed waves dominated by swell, in which the low-frequency peak of the spectrum is much larger than the high peak because the energy of the spectrum is concentrated in the low-frequency part. The second type is mixed waves dominated by wind wave, in which the highfrequency peak of the bimodal spectrum is larger than the low peak because the energy is concentrated in the highfrequency part. The last type is mixed waves with similar intensities of swell and wind waves. In this case, the highand low-frequency peaks of the bimodal spectrum exhibit slight differences (Fig.1). The bimodal spectrum is commonly expressed in the form of single-peak spectrum superposition. This study adopts the bimodal spectrum formula fitted by Chi et al. based on the measured data from a large number of spectrum formulae [26]. The low- 
frequency part of the formula uses the Neumann spectrum expression with the sharpness factor as a parameter in accordance with the proposal of Guan et al. [18]. The highfrequency part adopts the widely used JONSWAP spectrum, which can be defined as

$$
\begin{aligned}
S(\omega) & =\frac{m_{01}}{\omega_{1}} P\left(\frac{\omega}{\omega_{1}}\right)^{-p} \exp \left\{-\frac{p}{q}\left[\left(\frac{\omega}{\omega_{1}}\right)^{-q}-1\right]\right\} \\
& +16 \beta_{j} \frac{m_{02}}{\omega_{2}}\left(\frac{\omega}{\omega_{2}}\right)^{-5} \exp \left[-1.25\left(\frac{\omega}{\omega_{2}}\right)^{-4}\right] \gamma^{\exp \left[-\left(\omega / \omega_{2}-1\right)^{2} / 2 \sigma^{2}\right]}
\end{aligned}
$$

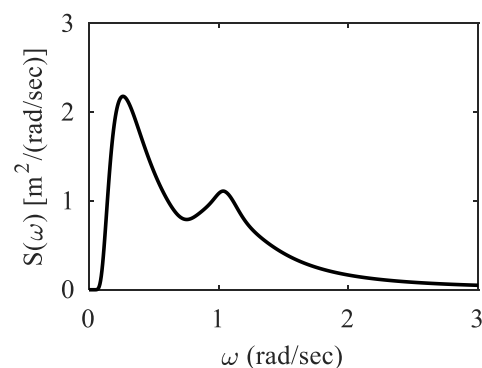

(a)

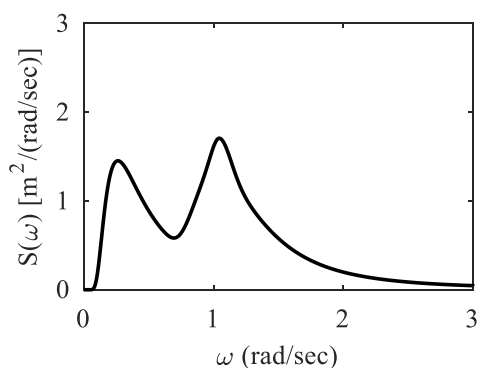

(b) where $P$ represents the sharpness factor, $p$ and $q$ are the spectral parameters $(p=e \cdot P+1, q=e \cdot P), e$ is the natural logarithm, $\omega_{1}$ and $\omega_{2}$ are the peak frequencies of the low and peak spectra, respectively, $m_{01}$ and $m_{02}$ are the zeroorder moments of the low and peak spectra, respectively, $\sigma$ is the peak shape coefficient ( $\sigma=0.07$ when $\omega \leq \omega_{2}$, whereas $\sigma=0.09$ otherwise), and $\gamma=1 \sim 7$ denotes the enhance factor, $\beta_{j}=\frac{0.06238(1.094-0.01915 \ln \gamma)}{0.230+0.0336 \gamma-0.185(1.9+\gamma)^{-1}}$.

Fig. 1. Bimodal spectral types. (a) Swell-dominated.(b) Mixed sea.(c) Wind wave-dominated

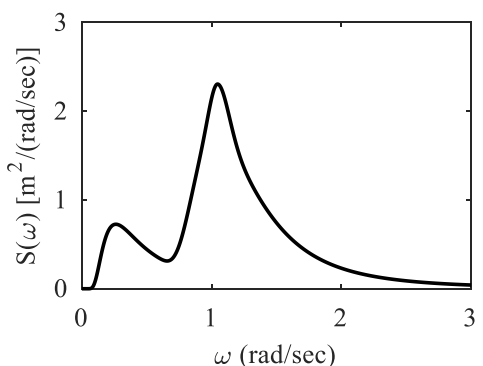

(c)

\subsection{Simulation of the second-order wave elevation}

In the present study, the wave elevation time history is obtained using the Monte Carlo simulation method. A second-order expansion of the sea surface can capture the effects of wave steepness, water depth, and directional spreading with no approximations other than the truncation of the expansion at the second order. The second-order wave interactions for infinite water depth are calculated using the Longuet-Higgins equation. These calculations were extended to intermediate water depth by Sharma and Dean [27]. It is reproduced the latter result for completeness. The first-order water surface is defined as

$$
\eta_{1}=\sum_{n=1}^{N} a_{n} \cos \left(\vec{k}_{n} x-\omega_{n} t+\varepsilon_{n}\right)
$$

where $t$ represents the time; $x$ is the position vector in the plane; $\omega_{n}, \varepsilon_{n}$, and $\vec{k}_{n}$ are the radian frequency, phase, and vector wave number of the Fourier wave $n$th component, respectively; and $a_{n}$ is the corresponding amplitude. The frequencies and wave numbers are related by the linear dispersion equation.

$$
\omega_{n}^{2}=g\left|\vec{k}_{n}\right| \tanh \left(\left|\vec{k}_{n}\right| d\right)
$$

where $g$ is the acceleration due to gravity and $d$ is the water depth. The second-order correction of the wave surface proposed by Sharma and Dean is expressed as

$$
\begin{aligned}
& \eta_{2}=\frac{1}{4} \sum_{i=1}^{N} \sum_{j=1}^{N} a_{i} a_{j}\left\{K^{-} \cos \left(\chi_{i}-\chi_{j}\right)+K^{+} \cos \left(\chi_{i}+\chi_{j}\right)\right\} \\
& K^{-}=\left[D_{i j}^{-}-\left(\vec{k}_{i} \cdot \vec{k}_{j}+R_{i} R_{j}\right)\right]\left(R_{i} R_{j}\right)^{-1 / 2}+\left(R_{i}+R_{j}\right) \\
& K^{+}=\left[D_{i j}^{+}-\left(\vec{k}_{i} \cdot \vec{k}_{j}-R_{i} R_{j}\right)\right]\left(R_{i} R_{j}\right)^{-1 / 2}+\left(R_{i}+R_{j}\right)
\end{aligned}
$$

$$
\begin{aligned}
& D_{i j}^{-}=\frac{\left(\sqrt{R_{i}}-\sqrt{R_{j}}\right)\left\{\sqrt{R_{j}}\left(\vec{k}_{i}^{2}-R_{i}^{2}\right)-\sqrt{R_{i}}\left(\vec{k}_{j}^{2}-R_{j}^{2}\right)\right\}}{\left(\sqrt{R_{i}}-\sqrt{R_{j}}\right)^{2}-k_{i j}^{-} \tanh k_{i j}^{-} d} \\
& +\frac{2\left(\sqrt{R_{i}}-\sqrt{R_{j}}\right)^{2}\left(\vec{k}_{i} \cdot \vec{k}_{j}+R_{i} R_{j}\right)}{\left(\sqrt{R_{i}}-\sqrt{R_{j}}\right)^{2}-k_{i j}^{-} \tanh k_{i j}^{-} d} \\
& D_{i j}^{+}=\frac{\left(\sqrt{R_{i}}+\sqrt{R_{j}}\right)\left\{\sqrt{R_{i}}\left(\vec{k}_{j}^{2}-R_{j}^{2}\right)-\sqrt{R_{j}}\left(\vec{k}_{i}^{2}-R_{i}^{2}\right)\right\}}{\left(\sqrt{R_{i}}+\sqrt{R_{j}}\right)^{2}-k_{i j}^{+} \tanh k_{i j}^{+} d} \\
& +\frac{2\left(\sqrt{R_{i}}+\sqrt{R_{j}}\right)^{2}\left(\vec{k}_{i} \cdot \vec{k}_{j}-R_{i} R_{j}\right)}{\left(\sqrt{R_{i}}+\sqrt{R_{j}}\right)^{2}-k_{i j}^{+} \tanh k_{i j}^{+} d} \\
& k_{i j}^{-}=\left|\vec{k}_{i}-\vec{k}_{j}\right| \\
& k_{i j}^{+}=\left|\vec{k}_{i}+\vec{k}_{j}\right| \\
& R_{i}=\left|\vec{k}_{i}\right| \tanh \left(\left|\vec{k}_{i}\right| d\right)=\omega_{i}^{2} x-\omega_{i} t+\varepsilon_{i},
\end{aligned}
$$

where $\varepsilon_{n}$ denotes the random phases uniformly distributed between 0 and $2 \pi$. In addition, $a_{n}=\sqrt{2 S_{\eta 1}\left(f_{n}\right)\left(\Delta f_{n}\right)}$, where $S_{\eta 1}$ is the one-sided power spectral density function associated with $\eta_{1}$ and $\Delta f_{n}$ is the frequency interval. $\eta_{1}$ represents the linear wave elevation, whereas the summation of $\eta_{1}$ and $\eta_{2}$ denotes the second-order nonlinear wave elevation. The nonlinear wave elevation is always skewed toward the positive $z$ direction (i.e., sharp peaks and flat 
troughs). This feature has been reported by many previous studies. The stronger the wave nonlinearity, the higher the level of skewness of the wave toward the peak.

Fig. 2 shows the duration of a second-order nonlinear wave simulation for a mixed wave. The energy occupied by the wind wave and swell is $75 \%$ and $25 \%$ of the total wave energy, respectively. The peak period of the main peak spectrum is $10 \mathrm{~s}$, and the water depth is $40 \mathrm{~m}$.

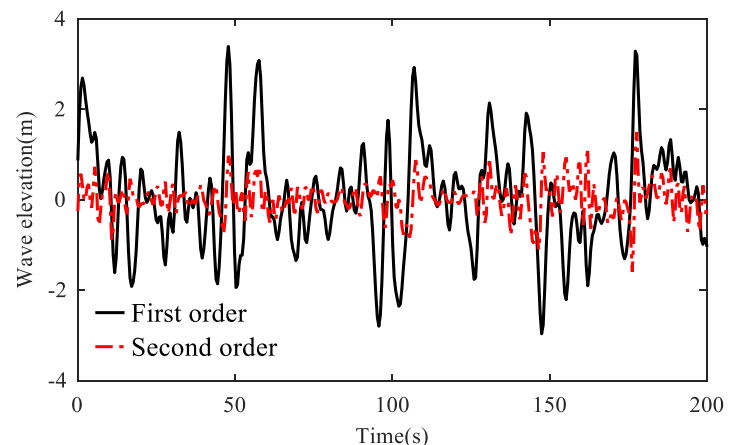

Fig. 2. Example of the wave for a double-peak spectrum with a peak period of $10 \mathrm{~s}$ and significant wave heights of 2.5 and $4.33 \mathrm{~m}$ at a water depth of $40 \mathrm{~m}$

\subsection{Spectral width and nonlinear coefficient}

Goda [28] suggested the use of the sharpness $Q_{p}$ to represent the narrowness of the spectrum.

$Q_{p}=\frac{2}{m_{0}^{2}} \int_{0}^{\infty} f\left[S_{\eta}(f)\right]^{2} d f$

Rye [29] stated that among many other parameters proposed to characterize the spectral width, $Q_{p}$ is the only factor that is not dependent on the high frequency cut-off choice. Goda reported $Q_{p}$ values obtained from singlepeaked spectra recorded in the Japanese coastal waters. The values range from 2 to 3. Fig. 3 presents the standardized JONSWAP spectrum. The $Q_{p}$ values associated with a JONSWAP wave spectrum with $\gamma=1$ and $\gamma=7$ are 2.008 and 4.6931, respectively. On the basis of this result, the smaller the value, the wider the spectrum.

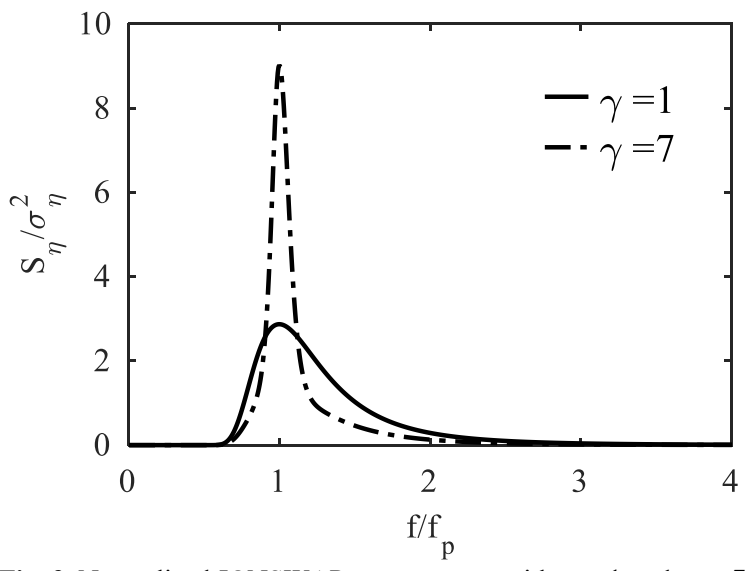

Fig. 3. Normalized JONSWAP wave spectra with $\gamma=1$ and $\gamma=7$
To measure the degree of nonlinearity of a random wave the term nonlinear parameter was introduced by Goda [30].

$\Pi=\left(H / L_{A}\right) \operatorname{coth}^{3}\left(k_{A} d\right)$

where $d$ is the water depth, $L_{A}$ is the wave length calculated using linear wave theory, and $k_{A}=2 \pi / L_{A}$ is the wave number. This nonlinear parameter becomes the wave steepness value in deep water and is close to Ursell number in shallow water.

When simulating the bimodal spectrum, we assume that the wave energy is conserved, that is, the significant wave height of a sea state is constant, whereas the swell and wind sea energy percentages vary. In this study, the swell percentages of the three spectral types account for $25 \%, 50 \%$, and $75 \%$, respectively. The simulation results show that the spectrum widths of the three wave spectra are different. Among them, the spectrum width of the swell-dominated spectrum is the largest, whereas that of the wind wave-based spectrum is the smallest; the latter is close to the single-peak spectrum value.

\section{Results and discussion}

\subsection{Effect of spectral width on wave height distribution}

This study simulates the second-order wave surface based on the method described in section 3.3. The wave height data are obtained through the upward zero-crossing method. The spectral width and nonlinear parameters of the spectrum are calculated in accordance with the approach introduced in section 3.4. For the convenience of comparison, the simulated bimodal spectrum maintains energy conservation, but the energy proportion of the swell and wind wave is different (section 3.2). Fig. 4 illustrates the effect of spectral width on wave height distribution. When the wave nonlinearity is extremely small (i.e., deep-water linear wave), the wave height predicted by the Rayleigh distribution is slightly larger in higher waves than in lower ones (Fig. 4(a)). Thompson [31], Haring et al. [32], Forristall [33], and Zhou [34] obtained the same conclusion. In addition, the spectral width has almost no effect on the wave height distribution under linear wave conditions.

However, as the level of wave nonlinearity increases, the influence of spectral width on wave height distribution becomes increasingly obvious. Fig. 4(b) depicts the case when $\Pi=0.0271$. The wave heights simulated in accordance with the wind wave-dominated and mixed sea types are close to the Rayleigh distribution. For the swelldominated sea state, a large spectral width results in the deviation of the wave height distribution from the Rayleigh distribution. Figs. 4(c) and 4(d) further demonstrate that as the level of wave nonlinearity increases, the influence of the spectral width on the wave height distribution intensifies. The larger the spectral width, the greater the deviation of the wave height distribution from the Rayleigh distribution. In such a case, the wave height predicted by the Rayleigh distribution is considerably small. The same phenomenon was observed by Forristall [11]. 


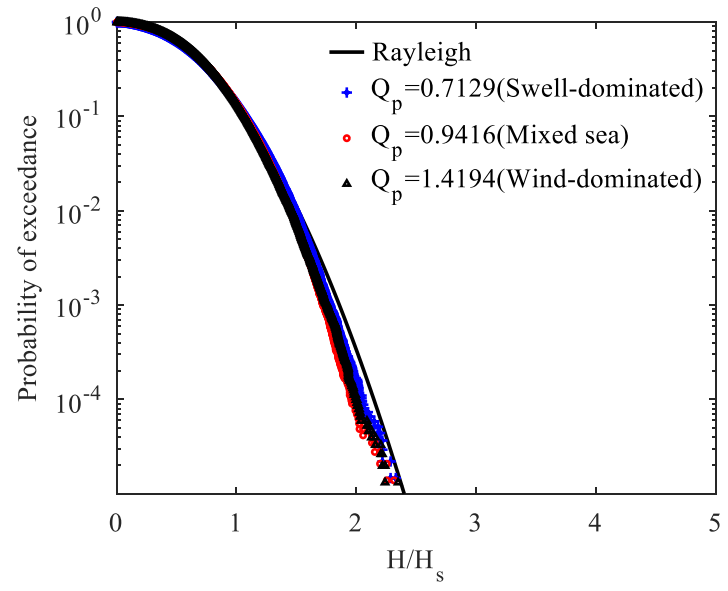

(a)

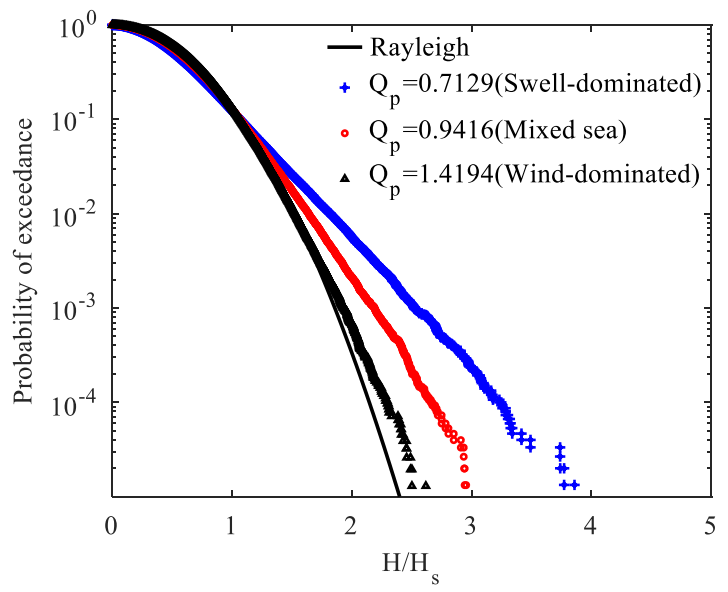

(c)

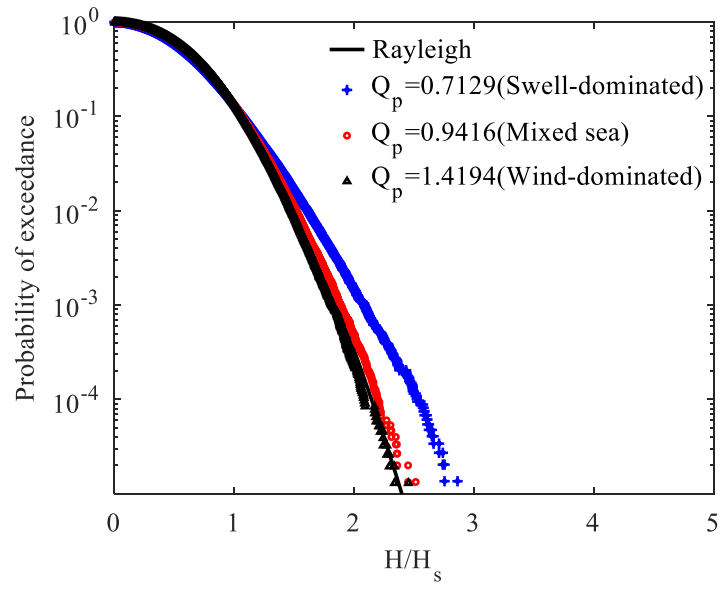

(b)

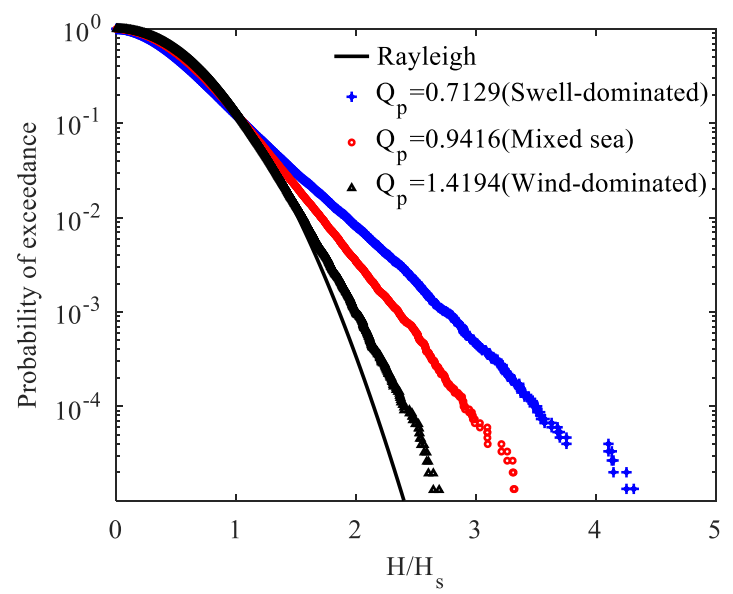

(d)

Fig. 4. Probability distribution of normalized wave heights with different spectral widths and similar nonlinearity. The wave heights are normalized by the significant wave height. (a) $\Pi=0.0142$. (b) $\Pi=0.0271$. (c) $\Pi=0.0463$. (d) $\Pi=0.0566 \mathrm{H}$

Fig. 4 also shows that when the wave nonlinearity is small, the spectral width exerts minimal small influence on the high-wave part. As the level of nonlinearity rises, the influence of the spectral width on the wave height distribution increases, especially in the medium and high wave ranges. In practical ocean engineering, engineers focus on high waves, which are closely related to the safety of the structures. Therefore, the effects of nonlinearity and spectral width on wave height must be considered.

\subsection{Effect of nonlinearity on wave height distribution}

The relationship between the wave height distribution and the nonlinear parameters under the same spectrum width is displayed in Fig. 5. As the level of nonlinearity increases, the wave heights of the swell-dominated waves obviously deviate from the Rayleigh distribution. When $\Pi=0.0566$, the once-in-a-thousand wave height can reach three times the significant wave height. When the swell and wind wave energies are equal and $\Pi=0.0271$, the wave height and Rayleigh distribution curves are the same. Similarly, the wave height distribution deviates from the Rayleigh distribution as the level of nonlinearity elevates. In this case, the once-in-a-thousand wave height can reach approximately 2.6 times the significant wave height. Given their small spectral width whose spectral type is the same as that of the narrow spectrum, the waves dominated by the wind sea exhibit the smallest difference between wave height and Rayleigh distributions. As the level of nonlinearity in the high-wave part (e.g., greater than the once-in-a-hundred-year wave height) increases, the wave height that is originally smaller than the Rayleigh distribution exceeds the latter. However, the degree of deviation is not large. The wave height increases with the increase in the level of nonlinearity because the wave steepness gradually rises due to wavewave interaction and shoaling. Forristall [11] obtained the same inference. Fig. 5 shows that when the spectrum width is small (i.e., similar to a narrow spectrum), the wave height distribution is similar to the Rayleigh distribution. A difference is observed in the extreme wave part, and the effect of nonlinearity on the wave height at this instance is small. When the spectral width is large, the nonlinearity substantially influences the wave height distribution. As the spectral width and level of nonlinearity increases, the deviation from the Rayleigh distribution increases from medium wave heights to high ones, especially for the parts with small probability. This phenomenon occurs because when random wave train propagates in shallow water, the wave-wave interaction and shoaling becomes noticeable. At this time, the wave crest becomes steep and narrow, whereas the wave trough becomes flat and wide. The wave steepness and wave height gradually increase.

When $\Pi=0.0566$ and the spectral width is at maximum, the value of $H / H_{s}$ is 4.4 (Fig. 4(d)). When $\mathrm{Q}_{\mathrm{p}}=0.7129$ and $\Pi=0.0566, H / H_{s}$ can reach a value of 5.0 (Fig. 5(a)). This result shows that under the same energy condition, 
Xiaolin Huan, Shifeng Xue, Xuejun Fan and Changliang Li/

Journal of Engineering Science and Technology Review 13 (6) (2020) 37 - 45

nonlinearity has a greater influence on the wave height distribution than the spectral width.

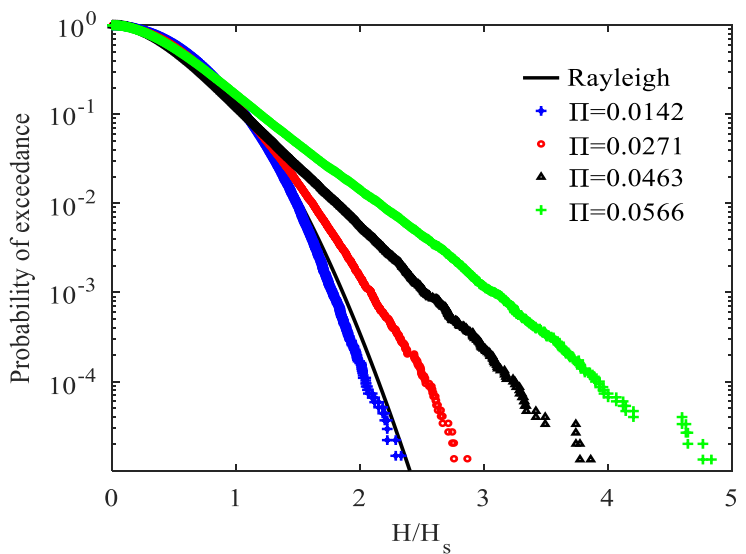

(a)

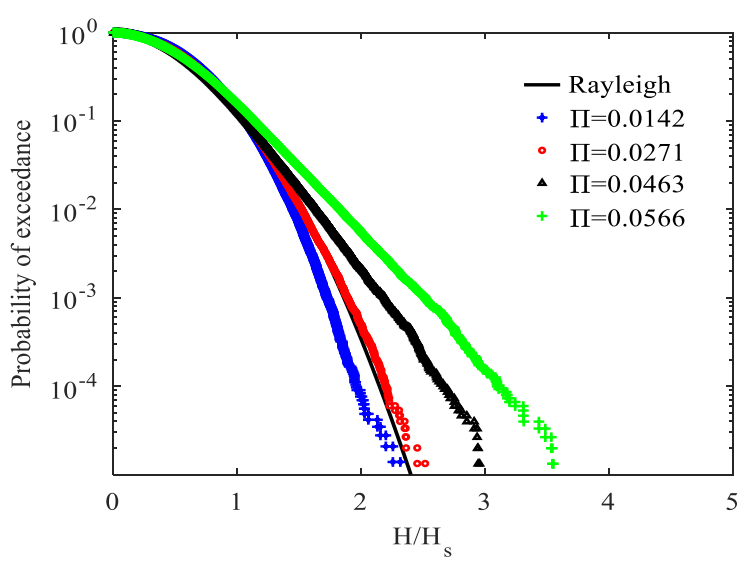

(b)

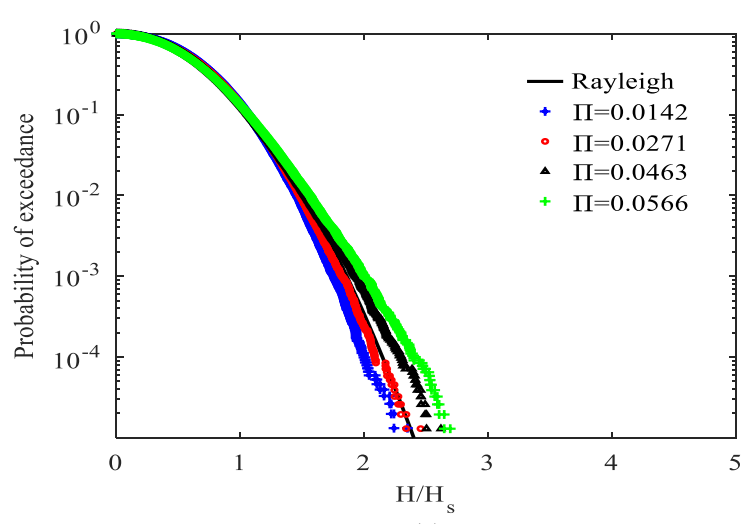

(c)

Fig. 5. Probability distribution of normalized wave heights with different nonlinearity levels and the same spectral width. The wave heights are normalized by the significant wave height. (a) $Q_{p}=0.7129$

(swell-dominated type). (b) $Q_{p}=0.9416$ (mixed sea type). (c) $Q_{p}=1.4194$ (wind-dominated type)

\subsection{Calculation of the Weibull distribution shape parameter}

On the basis of the above analysis, the spectral width and nonlinearity play important roles in wave height distribution. The traditional analyses of wave height distribution disregard both factors or consider only one of the two. Therefore, some scholars derived several analytical and experiential distributions that include the effect of wave steepness. However, these distribution formulae are rarely used because they are highly complicated, and their parameters are not easy to achieve. The Weibull distribution is relatively simple, with $\alpha$ as the most important factor (section 3.1). As previously established, $\alpha$ is a function of $\Pi$ and $Q_{p}$. In this study, a series of tests are performed through numerical simulation. By selecting the appropriate $\alpha$ value, a Weibull curve that fits the real wave height distribution can be obtained. This curve can be referred to as the new Weibull formula. The larger the spectral width and the higher the nonlinearity level, the lower the value of $\alpha$ (Fig. 6). Table 1 lists different values of $\alpha$ with various $Q_{p}$ and $\Pi$.

By using the MATLAB curve fitting technology, a fitting formula based on the calculated value of $Q_{p}$ and $\Pi$ can be obtained as

$$
\alpha=1.957-15.95 \times \Pi+0.5326 \times \mathrm{Q}_{\mathrm{p}}+104.6 \times \Pi^{2}-1.336 \times \Pi \times \mathrm{Q}_{p}
$$

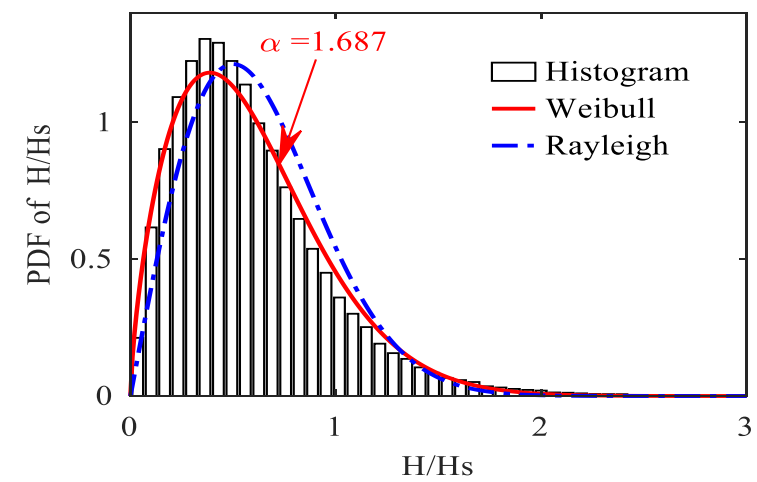

(a)

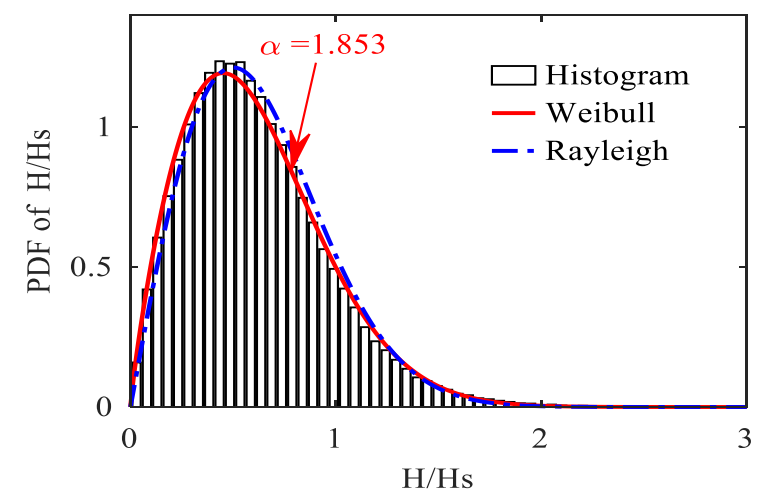

(b)

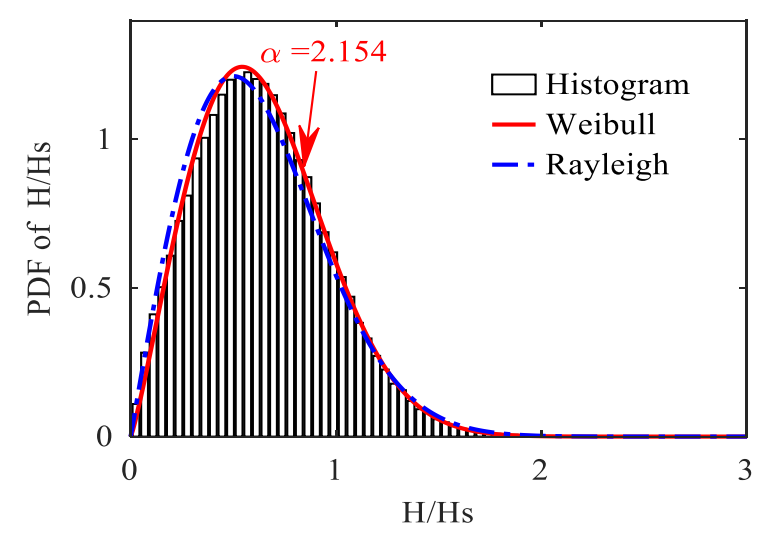

(c)

Fig. 6. Wave height PDF for different nonlinearity and spectral width parameters. (a) $\Pi=0.0463, Q_{p}=0.7129$. (b) $\Pi=0.0463, Q_{p}=0.9416$. (c) $\Pi=0.0271, \mathrm{Q}_{\mathrm{p}}=1.4194$ 
Xiaolin Huan, Shifeng Xue, Xuejun Fan and Changliang Li/

Journal of Engineering Science and Technology Review 13 (6) (2020) 37 - 45

Table 1. Numerical results of the shape parameters with different nonlinearity and spectral width values

\begin{tabular}{r|c|c|c|c|c}
\hline & & & & & \\
\hline 0.7129 & 2.051 & 1.895 & 1.687 & 1.554 & 1.392 \\
0.9416 & 2.204 & 2.103 & 1.853 & 1.754 & 1.621 \\
1.4194 & 2.256 & 2.154 & 2.087 & 2.043 & 1.952 \\
\hline$\Pi$ & & & & & \\
$\mathrm{Q}_{\mathrm{p}}$ & 0.0513 & 0.0544 & 0.0644 & 0.0739 & 0.0857 \\
\hline 0.7632 & 1.972 & 1.856 & 1.782 & 1.732 & 1.695 \\
0.9416 & 2.105 & 1.822 & 1.823 & 1.732 & 1.703 \\
1.2306 & 2.215 & 2.016 & 1.902 & 1.811 & 1.713 \\
\hline
\end{tabular}

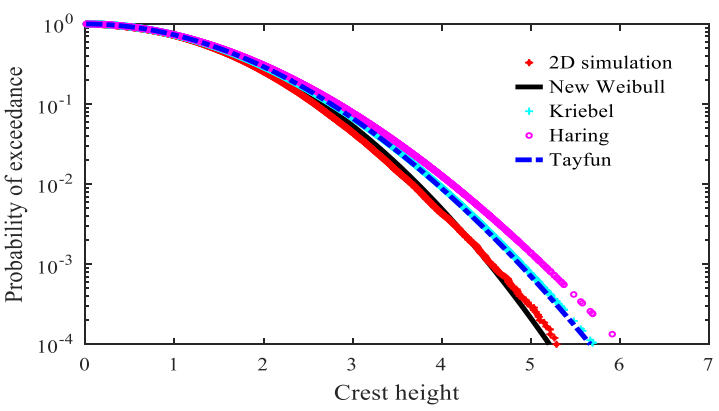

Fig. 7. Comparison of the crest height distribution when $D=60 \mathrm{~m}$, $T_{P}=15 \mathrm{~s}$, and $H_{s}=5.0 \mathrm{~m}$

\subsection{Formula verification}

To verify the accuracy of the proposed shape parameter fitting formula, Fig. 7 shows the comparison of the crest heights based on the second-order nonlinear simulation with the new Weibull distribution and the previous results obtained by Kriebel and Dawson [35], Haring et al. [32], and Tayfun [9]. The error between the theoretical formula and the simulated value is expressed in terms of the root mean square error.

$$
\mathrm{R}=\sqrt{\sum_{i=1}^{n}\left(p_{i}-s_{i}\right)^{2} / n}
$$

where $\mathrm{R}$ is the square root error, $p_{i}$ is the theoretical value, $\mathrm{s}_{i}$ is the simulated value, and $n$ is the sample size. The square root errors of the four distributions are $\mathrm{R}_{\text {Weibull }}=0.0291, R_{\text {Kriebel }}=0.0433, \mathrm{R}_{\text {Haring }}=0.0807$, and
$\mathrm{R}_{\text {Tayfun }}=0.0410$. The findings indicate that the proposed new Weibull distribution demonstrates the best fit of the simulation data.

\section{Conclusions}

To explore the characteristics of real ocean environment, a numerical method based on Monte Carlo approach was developed to simulate second-order nonlinear random waves and investigate the effects of the spectral width of different types of bimodal spectra and nonlinearity on wave height distribution. The following conclusions were drawn.

(1) In deep water conditions, the spectral width has minimal effect on the wave height distribution, and the wave height can be approximated as a Rayleigh distribution.

(2) When the wave nonlinearity is large, the Rayleigh distribution underestimates the wave height, especially the medium and high wave parts, as the spectral width increases. Under the energy-conserved condition, nonlinearity exerts a greater influence on wave height distribution than the spectral width.

(3) The influence of the spectral width and nonlinearity on the wave height distribution can be reflected by the fitted shape parameters of the Weibull distribution. The fitting formula is simple and accurate.

By combining theoretical analysis and numerical research, this study proposes a new method for calculating the design wave height. The distribution model established for nonlinear mixed waves is simplified and conforms to many sea conditions, and thus can serve as reference for coastal and ocean engineers. However, due to the lack of onsite and experimental data, the application of the proposed method is limited. Future studies will combine experimental data with this model and apply corrections to increase the accuracy and application range of the calculation of the design wave height.

\section{Acknowledgements}

This work was supported by the National Key Research and Development Program (No: 2016YFC0303801).

This is an Open Access article distributed under the terms of the Creative Commons Attribution License

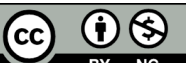

\section{References}

1. Longuet-Higgins, M.S., "On the Statistical Distribution of the Heights of Sea Waves". Journal of Marine Research, 1952, XI(3),pp.245-266.

2. Ding, K., Feng, X., Ma, G.F., "Study on the influence of wave nonlinearity on the wave penetration characteristics of slope dikes". Water Transport Engineering, 2020, 4, pp.1-8.

3. Feng, Q.D., Li, Z.G., Zhang, F., "Research on nonlinear wave loads and overtopping wave loads of ships in severe sea conditions". Ship Mechanics, 2019, 12, pp.1417-1424.

4. Zhou, Z.L., Zhang, H., Shi, Y.L., "Research on Nonlinear Characteristics of Nearshore Water Waves". Journal of University of Chinese Academy of Sciences, 2019, 4, pp.433-443.

5. Winston, G., "Wave Bimodal Spectrum based on Swell and Windsea Components". IFAC-Papers Online, 2015, V48(16),pp.223228.

6. Shao, Z.X., Liang, B.C., Li, H.J., "Extreme significant wave height

of tropical cyclone waves in the South China Sea". Natural Hazards and Earth System Sciences, 2019, V19(10), pp.2067-2077.

7. Kvingedal, B. ,Bruserud, K., Nygaard, E., "Individual wave height and wave crest distributions based on field measurements from the northern North Sea". Ocean Dynamics, V68(12), pp.1727-1738.

8. Mackay, E, Johanning, L., "Long-term distributions of individual wave and crest heights". Journal of Ocean Engineering, 2018, V165, pp.164-183.

9. Tayfun, M.A., "Narrow-band nonlinear sea waves". Journal of Geophysical Research, 1980,85(C3), pp.1548-1552.

10. Zhang, X.Y., Han, J., Wang, X., "Research on wave prediction method based on nonlinear Schrodinger equation". Acta Oceanologica Sinica, 2019, 11, pp.15-24.

11. Forristall, G. Z., "Wave crest distributions: observations and second-order theory". Journal of Physical Oceanography, 2000, 30 , pp.1931-1943.

12. Feng, S.Y., Ma, X.Z., Dong, G.H., "Research on the numerical model of high-order spectrum of wave height nonlinear probability distribution". Acta Oceanologica Sinica,2019,3,pp.44-51.

13. Andreas, F., Haselsteiner, K.T., "Predicting wave heights for marine design by prioritizing extreme events in a global model". Renewable Energy, 2020, 156, pp.1146-1157. 
Xiaolin Huan, Shifeng Xue, Xuejun Fan and Changliang Li/

Journal of Engineering Science and Technology Review 13 (6) (2020) 37 - 45

14. Li, X., Zhao, Y.N., Jin, H.P., "Wave height interval distribution method based on nonlinear shallow water wave funtion". Journal of Coastal Research, 2020,103,pp.843-846.

15. Tur, R., "Maximum wave height hindcasting using ensemble linearnonlinear models". Theoretical and applied Climatology, 2020, V141(3), pp.1151-1163.

16. Chen, W.W., Liu, X.L., Hang, Z., “Applicability of Ochi-Hubble doublet spectrum in the sea area near islands and reefs". China Shipbuilding, 2020, 1, pp.120-130.

17. Stephen, O., Harshinie, K., Dominic, E. R., "Effects of swell on wave height distribution of energy-conserved bimodal seas". Journal of Marine Science and Engineering, 2019, V7 (3), pp.1-16.

18. Guan, C.L., Zhang, D.C., Guan, S.Y., "A representation of double peak ocean wave spectrum”. Ocean and Limnology, 1996, 27(2), pp.151-156.

19. Forristall, G.Z., "Joint wave height and period statistics from linear simulations". Journal of Ocean Engineering, 2017, 3, pp.221-231.

20. Didenkulova, E.G., Slunyaev, A.V., Pelinovsky, E.N., "Numerical simulation of random bimodal wave systems in the $\mathrm{KdV}$ framework". European Journal of Mechanics/B Fluids, 2019, 78, pp.21-31.

21. Huang, W.N., Dong, S., "Joint distribution of individual wave heights and periods in mixed sea states using finite mixture models". Journal of Coastal Engineering, 2020, 161, pp.1-13.

22. Hu, S.-L J., "Nonlinearity and spectral width effects on ocean wave height distribution". In: $15^{\text {th }}$ ASCE Engineering Mechanics Conference, New York, USA: ASCE, 2002, pp.135-142.

23. Li, C.L., Liang, B.C., Zhao, L., "Dependence of shape parameter of weibull distribution on wave spectral width and nonlinearity". In: $27^{\text {th }}$ international conference on offshore mechanics and arctic engineering, Estoril, Portugal:ASME, 2008, pp.115-122.

24. Martell-Dubois, R., Silva-Casarin, R., Mendoza-Baldwin, E.G., "Spectral bimodality of waves produced by hurricanes in the Caribbean coastal zone off Mexico". Ciencias Marinas, 2018,V44(1), pp.33-48.
25. Alessandro, T., Miguel, O., Jaak, M., "Wave statistics in unimodal and bimodal seas from a second-order model". European Journal of Mechanics/B Fluids, 2006, 25, pp.649-661.

26. Chi, S.C, Tao, A.F., Li, X.D., "Research on the standard spectrum of double peaks based on measured data". Bulletin of Ocean Limnology, 2020, 1, pp.9-16.

27. Sharma, N.J., Dean, R.G., "Second-order directional seas and associated wave forces". Journal of Society Petroleum Engineering, 1980, pp.129-140.

28. Goda, Y., "Numerical experiments on wave statistics with spectral simulation". Report of Port and Harbor Research Institute, 1970, 9(3), pp.3-57.

29. Rye, H., "The stability of some currently used wave parameters", Journal of Coastal Engineering, 1977, 1, pp.17-30.

30. Goda, Y., "Random wave and spectra". In: Herbich J.B.Ed. Handbook of Coastal and Ocean Engineering, Houston, USA:GULT, 1990, V1, pp.175-212.

31. Thompson, E.F., "Results from CERC wave measurement program". In: Proceedings of the International Symposium on Ocean Wave Measurement and Analysis, New Orleans, USA: ASCE, 1974,1, pp.824-836.

32. Haring, R. E., Osborne, A. R., Spencer, L. P., "Extreme wave parameters based on continental shelf storm wave records". In: Proceedings of 15th Coastal Engineering Conference, Hawaii, USA: ASCE, 1976, pp. 151-170.

33. Forristall, G.Z. "On the statistical distribution of wave heights in a storm”. Journal of Geophysical Research, 1978,83, pp.2353-2358.

34. Zhou, L.M., Guo, P.F., Wang, Q., "Application of maximum entropy principle to study ing the distribution of wave heights in a random wave field". China Ocean Engineering , 2004,18(1), pp.6978.

35. Kriebel, D.L., Dawson, T.H., "Nonlinearity in crest height statistics". In: Proceedings of the Second International Conference on Wave Measurement and Analysis, New York, USA:ASCE, 1993 pp.61-75. 